\title{
JPEG, JPEG2000 and PBCS based Image Compression: An Experimental Analysis
}

\author{
Jayavrinda \\ Vrindavanam \\ Ph D student, Dept. \\ of E\&C, NIT, Durgapur
}

\author{
Saravanan \\ Chandran \\ Asst. Professor \\ Head, Computer Centre \\ NIT, Durgapur
}

\author{
Gautam K \\ Mahanti \\ Professor \\ Dept. of E\&C \\ NIT, Durgapur
}

\author{
Vijayalakshmi K \\ Sr.Lecturer \\ Dept of E\&C \\ Caledonaian \\ College, Muscat
}

\begin{abstract}
The paper attempts a comparison between Joint Picture Expert Group (JPEG), JPEG2000 and the novel method of position based coding scheme (PBCS) introduced by the authors, based on the output from different images. The results have proved that the proposed method is superior in terms of image compression ratio, PSNR and visual quality. After a review of various image compression standards and image compression coders, it is observed that there is a need to study the posttransformation matrix in a JPEG environment and accordingly, brought out a coding scheme based on the position of elements of the transform coefficients matrix after performing quantization. By identifying the unique elements and by reducing redundancies, the paper presents a novel method of coding called, PBCS. Thereafter, the results of JPEG, JPEG2000 with Huffman coding and PBCS are compared. The results show better compression ratio with higher PSNR and better image quality without quantization. The study can be considered as a logical extension of the image transformation matrix, applies statistical tools to achieve the novel coding scheme. The coding scheme can highly economise bandwidth without compromising picture quality; invariant to the existing compression standards, lossy as well as lossless compressions, which offers possibility for wide ranging applications.
\end{abstract}

\section{General Terms}

Image compression, Huffman coding, low bit rate transmission, JPEG, JPEG 2000, wavelet, PSNR, PBCS.

\section{Keywords}

JPEG, JPEG2000, image compression, wavelet, DCT, DWT, PBCS.

\section{INTRODUCTION}

It is well documented in the literature that compressed image transmission economizes bandwidth and therefore, ensures cost effectiveness. This has been the key driving factor of the ongoing research [1]. The major image compression techniques are JPEG standard and JPEG2000 [2,7]. JPEG image compression standard defines three different coding systems, such as loss baseline coding system based on DCT, an extended coding system for greater compression, higher precision, or progressive reconstruction of applications and lossless independent coding system for reversible compression [6]. At the application of JPEG, it is observed that the DCT leads to discontinuities at the boundaries of the 8 by 8 blocks. The colour of a pixel on the edge of a block can be influenced by that of a pixel anywhere in the block, but not by an adjacent pixel in another block. Further, the JPEG algorithm allows recovering the image at only one resolution. At times, it may be desirable to recover the image at lower resolutions, allowing the image to be displayed at progressively higher resolutions while the full image is downloaded. As advancements, the latest JPEG2000 standard encompasses not only new compression algorithms, but also flexible compression architectures and formats. JPEG2000 addresses areas where current standards have limitations in producing the best quality or performance and provides low bit rate operation (below 0.25 bits/pixel) with subjective image quality performance superior to previous standards without sacrificing performance at higher bitrates. The key differentiator is that the JPEG2000 uses a wavelet transform in place of DCT. JPEG2000 is based on DWT, which is applied on image tiles. DWT tiles are decomposed into different decomposition (resolution) levels [3, 4, 5]. After transformation, all transform coefficients are quantized. Scalar quantization is used in Part I of the standard. Arithmetic coding is employed in the last part of the encoding process.

As has been evident from the advancements as per the post JPEG2000 literature, despite considerable researches taking place in this specific field, scope still exists to explore alternative approaches for further image compression. The authors have postulated a novel algorithm by analyzing the hitherto unexplored segment, i.e., position of coefficients, through identification of common coefficients in order to achieve better compression results. This exercise, as it further analyses the transformation matrix can be regarded as a direct extension study of the wavelet based transform coefficient matrix. The study attempted to aggregate similar coefficients at the compression level and disaggregation at the decompression stage, as an alternative method, which we shown to ensure better image compression within the ambit of the existing wavelet methodology.

Since the focus of this paper is comparison between JPEG, JPEG2000 and the PBCS, the latter coding scheme is explained in detail as it is a novel method. The second section of this paper reviews the major developments in the field. The third section of the paper details the position based coding scheme and its methodology. Analysis and results of the proposed encoder based system in comparison with the existing image compression standards is attempted in the fourth section. The last section is by way of conclusion.

\section{REVIEW OF IMAGE CODING TECHNIQUES}

There are several coding schemes available in the literature, according to the applications and requirements. The feature of the lossless compression technique is that the original image can be perfectly recovered from the compressed image [2]. It is also known as entropy coding since it use decomposition techniques to eliminate or minimize redundancy. Lossless compression is mainly for applications like medical imaging, where the quality of image is important. Different coding systems follow different methods. In the case of Run length 
encoding, compression is performed by counting the number of adjacent pixels with the same gray-level value. This count, called the run length, is then coded and stored [8]. The number of bits used for the coding depends on the number of pixels in a row: If the row has $2^{\mathrm{n}}$ pixels, then the required number of bits is n. A $256 \times 256$ image requires 8 bits, since $2^{8}=256$. The other most commonly used lossless compression coding, is Huffman coding. The basic idea of Huffman coding algorithm was to assign shorter code words to more frequently used symbols. Huffman coding can generate a code that is as close as possible to the minimum bound, the entropy [5]. This method results in variable length coding. For complex images, Huffman code alone will reduce the file size by $10 \%$ to $50 \%$. By removing irrelevant information first, file size reduction is possible. In the case of LZW coding (Lempel-Ziv-Welch), coding can be static or dynamic, which is a dictionary based coding. In static dictionary coding, dictionary is fixed during the encoding and decoding processes. On the other hand, in dynamic dictionary coding, the dictionary is updated on fly. The computer industry is widely using LZW coding. It is also implemented as compressed command on UNIX. On the other hand, area coding is an enhanced form of run length coding, which reflects the two dimensional character of images. It is a significant advancement over the other lossless methods. It does not make much of a meaning to interpret the coding of an image as a sequential stream, as it is in fact an array of sequences building up a two dimensional object. The idea was to find the rectangular regions with the same characteristics. These rectangular regions are coded in a descriptive form as an element with two points and a certain structure. Area coding is highly effective and it can give high compression ratio but the limitation is non-linear in nature, which prevents the implementation in hardware.

The segment of lossy compression technique provides higher compression ratio than lossless compression. In this method, the compression ratio is high; the decompressed image is not exactly identical to the original image, but close to it. Since the quality requirements of the reconstructed image vary across different applications, different types of lossy compression techniques are widely used. The quantization process applied in lossy compression technique results in loss of information. After quantization, entropy coding is done like lossless compression. The decoding is a reverse process. The entropy decoding is applied to compressed data to get the quantized data. Dequantization is applied to it \& finally the inverse transformation is performed to get the reconstructed image. The compression performance is evaluated with the factors like Compression ratio, SNR (Signal-to-Noise Ratio) and Speed of encoding \& decoding [2]. The recent studies have also examined image compression algorithms based on the efficient construction of wavelet coefficient lower trees[9]. The study apart from grouping the coefficients, a fast way of coding them was also introduced. Here fast execution is achieved by means of a simple two-pass coding and one-pass decoding algorithm.

By taking note of the [10] property of excellent visual quality and compression rate of fractal image coding, a recent paper presented a new fast and efficient image coder that applied the speed of the wavelet transform to the image quality of the fractal compression. Here encoding using Fisher's domain classification is applied to the Low pass subband of the wavelet image and a modified set partitioning in hierarchical trees coding, on the remaining coefficients. The proposed scheme has an average of $94 \%$ reduction in encoding decoding time comparing to the pure accelerated fractal coding results. In yet another paper, [11] an iterative algorithm was proposed, which the authors argue that, not only results in a compressed bit stream completely compatible with existing JPEG and MPEG decoders, but also computationally efficient when tested over standard test images. It achieves the best JPEG compression results to the extent that its own JPEG compression performance even exceeds the quoted PSNR results of some state of the art wavelet based image coder such as Shapiro's embedded zero tree wavelet algorithm at the common bit rates under comparison. Both the graph based algorithm and the iterative algorithm can be applied to application areas such as web image acceleration, digital camera image compression, MPEG frame optimization and transcoding etc. A new similarity measure for fractal image compression was introduced in another study [14]. When the original image is corrupted by noises, the authors have argued that the fractal image compression scheme should be insensitive to those noises presented in the corrupted image, as the underlying premise is it utilizes the self-similarity property in the image to achieve the purpose of compression. In order to overcome the high computational cost, the authors have applied the search technique of particle swarm optimization technique. The authors demonstrated that the proposed HFIC is robust against others in the image unlike the least square based regression technique. It can also reduce the encoding time while retaining the quality of the retrieved image.

A novel way of representing image information in a wavelet domain [12] was introduced to develop and analyze the 'spherical representation'. After a review of the existing wavelet based image properties and the weaknesses of the existing models, the authors suggest that wavelet subbands are best characterized by spatially varying non homogeneous processes. Since edges and texture come in arbitrary locations, orientations and shapes, information about the location of high activity areas allows the coding methods to be successfully adapted the statistics of different regions. The authors argued that parametric descriptions of wavelet coefficient distributions are especially prone to mismatches. The authors have formed a hierarchical tree of subband energy by summing up the squared coefficients and non-homogenous wavelet bands are handled through one of the non-parametric model of the hierarchy. A review of the statistical based image compression scheme [13], confirmed that the wavelet based encoders have proven ideally suited for embedding compete human visual system models on account of the space frequency localization properties of wavelet decompositions. The authors have evolved a wavelet based image compression scheme, which employs a novel statistical encoding algorithm and the properties of the human visual system to code the wavelet coefficients in each subband. The coefficients in each subband are coded using the statistical algorithm developed by the authors. The statistical encoder applied a hierarchical estimation algorithm to code the coefficients in each detailed subband, which assumes a Gaussian distribution in some regions. The coefficients in each subband were first shifted to have a minimum value of zero and then passed to the encoder along with a threshold value specifically generated for that subband. The results indicate that the coding scheme show significantly higher subjective and objective quality when perceptual weights is used to regulate the thresh hold values of different subbands. It also outperforms JPEG in higher compression ratios.

As evident from the recent advancements, the previous studies were essentially concentrated either on developing certain types of algorithms to address specific issues or to integrate certain systems within the domain to achieve certain practical results. From the perspective of position based coding 
scheme, it may be desirable to refer an important study carried out by Said and Pearlman [15], as an extension study of the EZW algorithm by Shapiro [16]. Said and Pearlman introduced a new algorithm called Set Partitioning in Hierarchical Tree (SPIHT); the distinguishing feature of the SPIHT is that the ordering data is not explicitly transmitted; this is based on the premise that the execution path of any algorithm is defined by the results of the comparisons on its branching points. In case both the encoder and decoder have the same sorting algorithm, then the decoder can duplicate the encoder's execution path based on the results of the magnitude comparisons and the ordering information can be recovered from the execution path. The concentration of the study was self similarity across different scales of image wavelet transform. As an extension to Shapiro's EZW algorithm, a recent study [17] proposed an image compression method with the support of wavelet transform, zero tree coding and adaptive arithmetic coding. The authors explored a novel static zeroth order adaptive arithmetic coder for better compression ratio. The method decomposed the image into several subband images using the discrete wavelet transform, de-correlated coefficients quantized by Shapiro's embedded zerotree wavelet algorithm and encoded using static zeroth order adaptive arithmetic coder. The results showed better compression ratio and reduced coding time.

The review makes it clear that, possibilities exist to explore further on the position of the transformation matrix. This is the context in which, the authors proposed the research study, which has postulated a novel statistical algorithm through identification of unique transform coefficients after quantization and position of the coefficients in the transform matrix in order to achieve better compression results. The study attempted aggregation of similar unique coefficients after performing quantization and their positions at the compression level and disaggregation at the decompression stage, as an alternative method. The paper compares the results of PBCS with standard image compression standards such as JPEG and JPEG2000 based on experimental results.

\section{POSITION BASED CODING SCHEME}

The basis of the alternative methodology goes back to the observation and analysis of the position of unique elements of 256X256 image post-wavelet transformation matrix. An analysis of the post-wavelet transformation matrices of different images carried out by the authors revealed that, there are repetitions of elements in such matrices. Logically, avoiding of such repetitions can contribute to higher image compression. As explained in the section on literature review, an analysis of the elements with a view to avoid repetitions in transmission matrix has not been attempted so far, possibly on account of the complexities in the post compression restoration process. Thus, the challenge is to ensure that the decoded matrix retains the same elements as that of the original post-wavelet transformation coefficients and also to ensure that only unique elements are transmitted. Thus, the objective has been to find out the unique elements as well as their position in order to avoid repetition of similar elements while transmission. Accordingly, a position coding scheme needs to be developed to address the above approach. Logically, such an approach would also be independent of existing transformations and quantization processes, which can be, therefore, applied in both lossy and lossless compression methods as the coding system is applied on the post-wavelet transformation matrix.

After performing such an analysis, the authors observed that, the compression ratio is not significantly reduced in comparison with the existing coding scheme including Huffman coding. The main reason for this is the size of the position elements, which is still a factor which may not yield better compression as we need to transmit position matrix and unique coefficient matrix with or without quantization for enabling the decoding process. Therefore, further reduction needs to be performed to reduce the matrix size keeping in view the ease of decoding without disturbing the restorability of unique positions. In order to reduce the size of the position elements, we have applied, the most commonly used measure of dispersion, standard deviation, to the position matrix. Standard deviation takes into account the dispersion of the elements from the mean of the matrix. With the support of mean and the standard deviation the reversal operation can also be performed without compromising on the position of elements. Since standard deviation takes the difference of elements with the mean, the resultant elements would be similar and also carries lesser bits to represent each number. Accordingly, for the reconstruction purpose, transmission of the reduced position matrix after applying standard deviation along with mean and unique coefficient matrix will be sufficient. This process can also be seen as a direct extension of the 256X256 post-transformation matrix, and accordingly, the coding scheme is simple to comprehend and works as logical extension to the wavelet transformation matrix. The results were found to be highly encouraging in comparison with the existing coding schemes. The test results of the position coding scheme as per this methodology offer better compression ratio without compromising on quality of the image and results into better PSNR.

The methodology explained above is demonstrated with the support of a process flow as given in the Fig 1 .
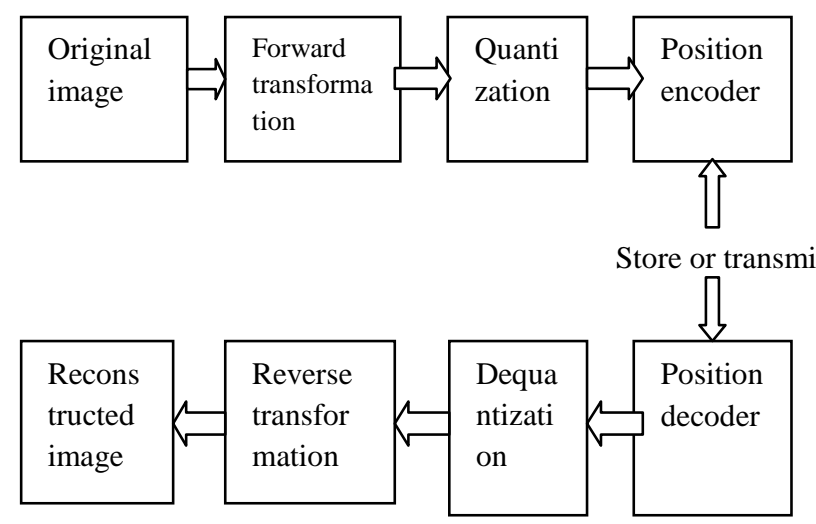

Fig 1: Position Based Image Compression

The original image is transformed by using DCT for JPEG and DWT for JPEG2000. Thereafter, encoding is performed with the support of the position based coding scheme(PBCS) encoder. The resultant output of the PBCS, such as position matrix after performing standard deviation, unique coefficients, and mean of position matrix are transmitted. At the receiving end inverse coding is applied and the original matrix is retrieved. Thereafter inverse transformation is applied to retrieve the original image. Further, in a position matrix, the values are always positive. This property gives the comfort to perform statistical operations on the position matrix, which has supported the use of standard deviation to generate the reduced position matrix. An illustration of the methodology is explained below. 
Let us take a $3 \mathrm{X} 4$ matrix as the sample post transformation matrix as shown below. From this matrix, we have created a unique coefficient matrix (E) and position matrix (P).

$$
\left[\begin{array}{lllll}
1 & 2 & 3 & 5 & \\
2 & 4 & 1 & 5 & \\
5 & 6 & 2 & 4
\end{array}\right]
$$

Out of the matrix shown above, matrix $\mathrm{E}$ is derived with unique elements. The positions of corresponding elements are reflected in $\mathrm{P}$.

$$
E=\left[\begin{array}{l}
1 \\
2 \\
3 \\
4 \\
5 \\
6
\end{array}\right]
$$

$\mathrm{P}_{\text {row }}=\left[\begin{array}{llllllllllll}1 & 2 & 1 & 2 & 3 & 1 & 2 & 3 & 1 & 2 & 3 & 3\end{array}\right]$

$\mathrm{P}_{\mathrm{col}}=\left[\begin{array}{llllllllllll}1 & 3 & 2 & 1 & 3 & 3 & 2 & 4 & 4 & 4 & 1 & 2\end{array}\right]$

Here, $\mathrm{P}_{\text {row }}$ represents the row position of the elements in E. $\mathrm{P}_{\text {col }}$ represents the column position of the elements in E. Suppose any value is repeating for entire rows of a particular column then that will be representing with special character and coded with minimum number of bits or $\mathrm{P}_{\text {row }}$ value will be represented with special character. After forming the row and column matrix, standard deviation is applied to both the matrices by taking the difference between the mean and the elements of the position matrices, shown below.

$\operatorname{Mean}\left(\mathrm{P}_{\text {row }}\right)=2, \operatorname{Mean}\left(\mathrm{P}_{\text {col }}\right)=2.5$

$\mathrm{P}_{\text {row }}=\left[\begin{array}{llllllllllll}1 & 0 & 1 & 0 & -1 & 1 & 0 & -1 & 1 & 0 & -1 & -1\end{array}\right]$

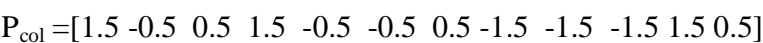

After taking the difference between the mean and the elements of the position matrix the authors found most of the values are similar and less number of bits are required to represent the elements of the position matrix. This will lead to more compression on position matrix. At the decoder side inverse operation is performed to get the position matrices and unique coefficient matrix. Also the coefficient of variation $(\mathrm{CV})$ is calculated to understand the degree of dispersion, as $\mathrm{CV}$ being an invariant measure, higher $\mathrm{CV}$ as a percentage shows higher degree of dispersion. Therefore, $\mathrm{CV}$ as a measure can also be compared with compression ratio and PSNR. Higher the CV, lower will be the compression ratio and lower $\mathrm{CV}$ will have higher compression ratio.

As a next step, the elements and the position of the elements are the input to the position decoder. At the decoder side, it decodes and as a reverse operation, gets the original wavelet coefficient matrix. Since no loss of information takes place during the coding process, the proposed method shall be categorized under lossless coding technique. Further, the PBCS simplifies the computation and makes it easily comprehensible. In order to derive the best results, the novel coding scheme presumably can be applied to images with lesser variations in colour or more similar coefficients as the size of the unique coefficient matrix can be logically at a reduced level. Since the novel approach is not probabilistic and lossless, it can also be used to environments where high quality image is of prime importance.

As has been evident from the process flow, the process makes quantization redundant and hence, it can be applied to any type of images where the quality is of prime priority. Since better compression ratio can be obtained without quantization, we have the range of options available taking into account enhanced compression ratio requirements and image quality.

\section{EXPERIMENTAL RESULTS}

In order to experiment PBCS, MATLAB software based programs were developed. This involves development of JPEG image compression, JPEG2000 compression with Huffman coding and the PBCS coding scheme. Huffman coding has been chosen for comparison as it supports lossless compression, as the proposed system can apply without quantization as a lossless compression. The authors have also performed a comparative analysis of all the three methods with quantization. The experiment has been conducted on several types of images for testing purposes with different colour schemes and background. The results in terms of compression ratio, $\mathrm{CV}$ and PSNR etc. are compared.

Table 1. Compression Ratio, CV and PSNR of PBCS Without quantization

\begin{tabular}{|c|c|c|c|}
\hline Images & $\begin{array}{c}\text { Compressi- } \\
\text { on Ratio }\end{array}$ & $\begin{array}{c}\text { Coefficient } \\
\text { of Variation }\end{array}$ & PSNR \\
\hline Fruits & $1: 8.7155$ & 188.38 & 36.1328 \\
\hline $\begin{array}{c}\text { Mara } \\
\text { Dona }\end{array}$ & $1: 8.4705$ & 193.59 & 36.1267 \\
\hline Lena & $1: 8.4223$ & 170.72 & 36.1221 \\
\hline Pisa & $1: 8.3325$ & 177.35 & 36.1218 \\
\hline Autumn & $1: 8.7717$ & 194.6 & 36.1387 \\
\hline Spring & $1: 8.149$ & 155.88 & 36.1083 \\
\hline
\end{tabular}

The compression ratio of the test images range between 81.49 to 87.71 . The variation can be explained through the CV. The correlation coefficient (r) is 0.53 , indicates a high positive correlation between compression ratio and CV. The CV has a range between $155 \%$ and $194 \%$. The $\mathrm{CV}$ being the ratio between the standard deviation and mean of the position matrix broadly explains as a measure the degree of dispersion within the matrix. Thus, it would be natural to find a higher degree of positive correlation between compression ratio and CV. Table 2 shows the comparison between JPEG2000 with Huffman coding and PBCS. Since PBCS compression ratio depends on the number of unique elements, the results show a fixed compression ratio. Hence the comparison is performed with a 
constant PSNR for both. The result shows that PBCS has better compression ratio than JPEG2000 with Huffman coding.

Table 2. Comparison Between Huffman and PBCS coding

\begin{tabular}{|c|c|c|c|}
\hline Images & $\begin{array}{c}\text { Compression } \\
\text { Ratio for } \\
\text { JPEG2000 } \\
\text { with PBCS }\end{array}$ & $\begin{array}{c}\text { Compression } \\
\text { Ratio for } \\
\text { JPEG2000 } \\
\text { with } \\
\text { Huffman }\end{array}$ & PSNR \\
\hline Fruits & $9.2891: 1$ & $5.981: 1$ & 27.2368 \\
\hline Maradona & $9.2916: 1$ & $7.5004: 1$ & 28.5572 \\
\hline Lena & $9.2935: 1$ & $7.5846: 1$ & 28.3660 \\
\hline Pisa & $9.2931: 1$ & $7.8208: 1$ & 28.6045 \\
\hline Autumn & $9.2925: 1$ & $5.6975: 1$ & 26.3748 \\
\hline Spring & $9.2891: 1$ & $8.4318: 1$ & 30 \\
\hline
\end{tabular}

An analysis is also attempted to explore the relationship between the Huffman coefficient and the unique coefficients produced by PBCS for the test images. For this purpose, the number of coefficients in the JPEG, JPEG2000 and the PBCS are calculated (Table 3). From the Table 3, it is clear that the unique coefficient size is smaller than Huffman DC and AC coefficient size.

For instance, the image 'autumn' has higher value of Huffman's AC coefficient than all other images and the compression rate per pixel is also higher for both JPEG and JPEG2000. The unique coefficient of PBCS has the lower size than the Huffman's coefficients. The size of the unique coefficients depends upon the types of images. An image with less dispersion will have relatively smaller number of unique coefficients and image with higher dispersion will have larger number of unique coefficients. The image 'Spring' has comparatively lower dispersion (it is clear from the $\mathrm{CV}$ value in Table 1) so the unique coefficient number is lower at 7976 . The proposed system will work well for the images with lower dispersion in the coefficient matrix. Accordingly the correlation coefficient ' $r$ ' between Huffman's AC coefficients of JPEG and PBCS unique coefficient coding scheme stands at 0.66 shows higher positive correlation. The correlation coefficient for the similar variables for JPEG2000 and PBCS stands at 0.50, which also shows similar higher positive correlation.

Table 3. Comparison between Huffman Coefficient and Unique Coefficient

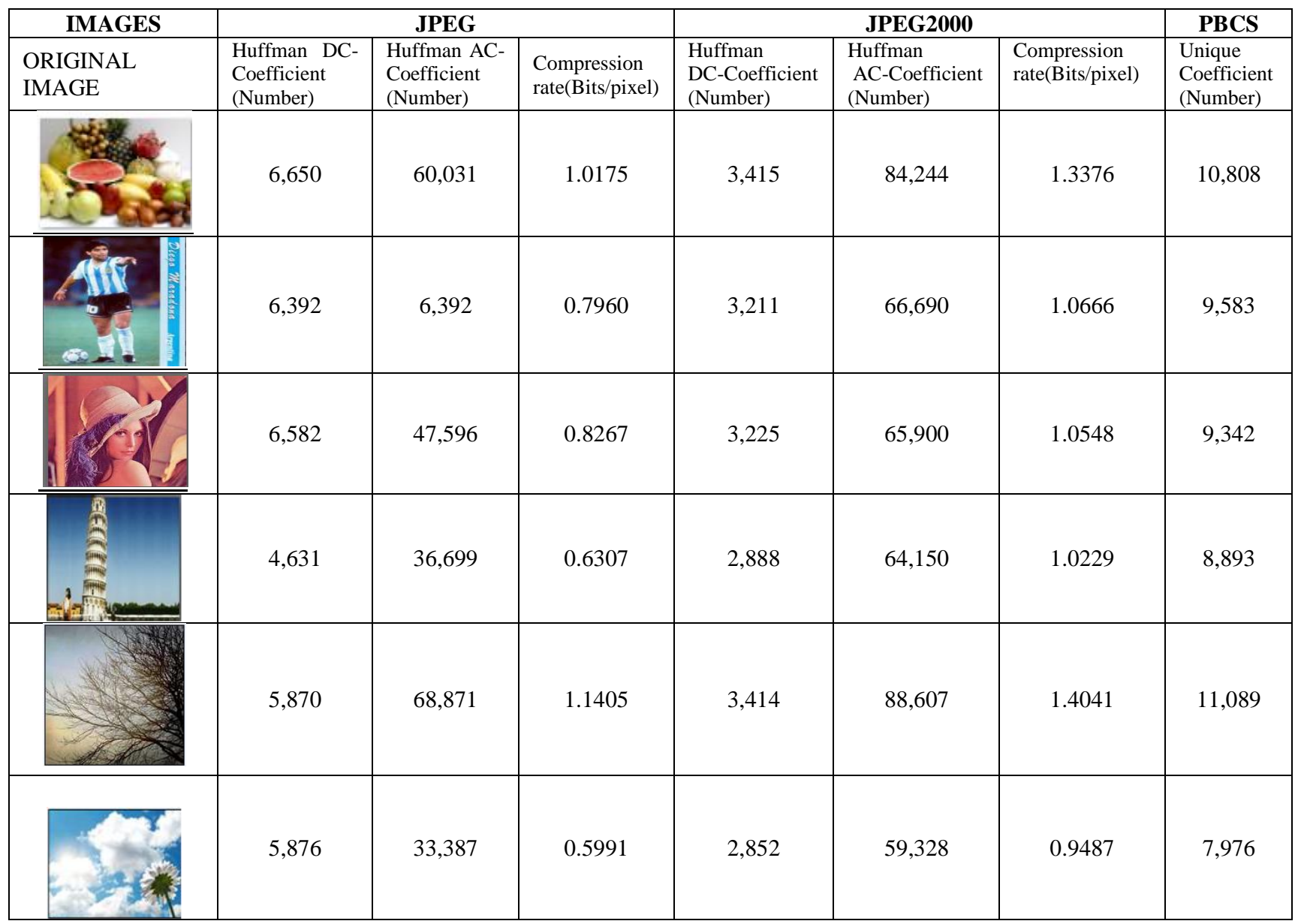




\section{CONCLUSION}

A study of coding schemes along with PBCS in the context of compression ratio, PSNR is presented in the paper. After a detailed analysis and observation of the existing coding schemes and image quality outputs, and also based on observed gaps in the existing coding schemes, the paper has attempted to exploit the repetitive values in transformation matrix, which until now continues to be an under researched area, for achieving better image compression with higher PSNR and image quality. Most importantly, the area of study features as a direct logical extension to the wavelet based approach and calculations are extremely simple to comprehend without adding complications in calculations. Since the authors did not use estimations for obtention of the PBCS, the resultant images are not impaired. This approach has several advantages, as it economizes the bandwidth apart from ensuring better image quality. Its quality of invariance to quantization makes it independent for use in different environments depending upon the requirement of image quality. This novel coding scheme can be applied to all segments in general and areas where precision is required in particular. As a next step forward, the authors would also be studying other alternatives in the similar domain to achieve better results.

\section{REFERENCES}

[1] Gonzalez R. C, E. R., and W.2008 Digital Image processing, New Delhi: Pearson Pentice Hall, Third Edition, Low price edition, Pages(1-904).

[2] Jayavrinda Vrindavanam, Chandran. S., and Mahanti, G. $\mathrm{K}$. 2012. A survey of image compression methods.;International Journal of computer application. Proceedings on International Conference and workshop on Emerging Trends in Technology (ICWET), March(pp.12-17), Mumbai, India.

[3] Jayavrinda Vrindavanam, Chandran. S., and Mahanti, G. K . 2012. Wavelet and JPEG based image compression: An experimental analysis.; International Journal of computer application. Proceedings on International Conference and workshop on Emerging Trends in Technology (ICWET), March(pp. 36-42), Mumbai, India.

[4] Poorva, Jayavrinda, V. 2007. "Low Bit rate Movie Transmission: An exploratory Analysis.; Proceedings of the Second International Conference on Industrial and Information Systems, Sri Lanka.

[5] Jayavrinda Vrindavanam. 2010. Video Compression for Movie Transmission: A Comparative Analysis.; Proceedings on International Conference and workshop on Emerging Trends in Technology (ICWET), Feb (pp. 173-175),Mumbai, India.[Digital.ACM 978-1-60558812-4].

[6] Sonja Grgic, M. M. 2001. Comparison of JPEG Image Coders.; Proceedings of the 3rd International symposium on Video Processing and Multimedia Communications, June, (pp. 79-85). Zadar, Croatia.

[7] ISO/IEC JTCI/SC29/WGI N1646R. 2000. JPEG 2000 Part I Final Committee Draft Version 1.0 March.

[8] Sonal, D. K. 2007. A study of various image compression techniques. COIT, RIMT-IET. Hisar.

[9] Oliver J. Malumbes, M. 2006. Low - Complexity Multiresolution Image Compression Using wavelet lower trees. IEEE Transactions on Circuits and Systems and Video Technology, Vo. No. 16, November.

[10] Cruz, Y. L. 2006. A Fast and Efficient Hybrid Fractal Wavelet Image Coder. IEEE Transactions on Image Processing, January, Vol 15, No.1.

[11] Longji, W. E.-h. 2009. Joint Optimization of Run-Length coding, Huffman coding and Quantization Table with Complete Baseline JPEG Decoder Compatibility. IEEE Transactions on Image Processing, Vol 18, No. 1, January.

[12] Hasan, F.A., Michael, T.2009 .Spherical Coding Algorithm for Wavelet Image Compression. IEEE Transactions on Image Compression, Vol.18, No. 5, pp. 1015-1024.

[13] Zadeh, P. B, A. S. A., and T. B., and Soraghan, J.2010. Multiresolution HVS and Statistically based Image Coding Scheme, Springer, ( pp. 347-370), Issue 49.

[14] Jeng, J. H., Tseng, C. C. and Hsieh, J. G. 2009. Study on Huber Fractal Image Compression," IEEE Transactions on Image Processing, (pp. 995-1003), vol. 18, no. 5.

[15] Said, A., Pearlman, W. A. 1996. A New,Fast, and Efficient Image Codec Based on Set Partitionaing in Hierarchical Trees. IEEE Transactions on Circuits and Systems for Video Technology, June, Volume 6, NO.3.

[16] Shapiro, J. M. 1993. Embedded image coding using zerotrees of wavelets coefficients. IEEE Transactions on Signal Processing, December(pp.3445-3462), Volume 41.

[17] Sulthana, N. S., Mahesh C. 2010. Image Compression with Adaptive Arithmetic Coding. International Journal of Computer Applications, February, 1(1):31-34, 